La deuxième étude nationale sur la gestion des risques cliniques (GRC) 2010 fait suite au projet de recherche "Gestion des risques cliniques dans les hôpitaux suisses» de I'EPF Zurich et de la Haute école de gestion de Lucerne (économie). Ce projet, axé sur la pratique, a notamment été suivi et cofinancé par la FMH.

Un monitorage continu et structuré est nécessaire pour mettre en évidence et soutenir la gestion des risques cliniques en Suisse. Le projet touchera bientôt à sa fin pour les instituts de recherche, mais on ne sait pas, à l'heure actuelle, qui se char- gera de poursuivre le monitorage. Une fois de plus, il serait regrettable qu'une initiative à peine commencée visant à garantir la qualité des soins et à promouvoir la sécurité des patients n'aboutisse pas. II serait beaucoup plus judicieux de mettre en œuvre et d'institutionnaliser l'instrument éprouvé - et par ailleurs également reconnu à l'étranger - que nous présentent ici les auteurs.

Martina Hersperger, responsable du département DDQ de la FMH

\title{
Zweites nationales Monitoring zum klinischen Risikomanagement im Spital
}

\section{Matthias Briner}

Zentrum für Organisations- und Arbeitswissenschaften, Forschungsgruppe «Psychologie der Arbeit in Organisation und Gesellschaft», ETH Zürich; IBR Institut für Betriebs- und Regionalökonomie, Hochschule Luzern - Wirtschaft

* In Zusammenhang mit diesem Projekt bereits erschienene Beiträge finden sich auf Seite 466 in den Literaturhinweisen $1,2,3$ und 9 .

Korrespondenz:

lic. phil. Matthias Briner Institut für Betriebs- und

Regionalökonomie IBR

Zentralstrasse 9

CH-6002 Luzern

Tel. 0412289939

matthias.briner@hslu.ch

www.rms.ethz.ch

\section{Die Studie in Kürze}

Das 2. nationale Monitoring zum kRM 2010 ist eine Folgestudie des Projekts «Klinisches Risikomanagement in Schweizer Spitälern» der ETH Zürich und der Hochschule Luzern - Wirtschaft (Projektbeschrieb in [1]). Im Rahmen des Projekts wurde ein MonitoringInstrument für kRM entwickelt [2]. 2007/2008 wurde es ein erstes Mal in der Schweiz eingesetzt mit dem Ziel, die aktuelle Situation und geplante Entwicklungen im kRM aufzuzeigen (Zusammenfassung der Resultate 2007/2008 in [3]). KRM wurde als die Gesamtheit der Strukturen, Prozesse, Instrumente und Aktivitäten definiert, welche die Mitarbeitenden eines Spitals unterstützen, die Risiken bei der Patientenversorgung $\mathrm{zu}$ erkennen, $\mathrm{zu}$ reduzieren und $\mathrm{zu}$ bewältigen (siehe auch [4]). Ausgehend von den Ergebnissen führten die Projektverantwortlichen im Juni 2009 eine gutbesuchte Transfertagung zum kRM durch (Unterlagen der Tagung finden Sie auf www. rms.ethz.ch). Dank des zweiten Monitorings stehen nun erstmals Daten von zwei Messzeitpunkten zum kRM in der Schweiz zur Verfügung, womit ein vertieftes Bild zur nationalen Lage im kRM entsteht. Die teilnehmenden Spitäler können ihre Angaben mit allen Spitälern und mit Spitälern ihres Spitaltyps vergleichen. Spitäler, die an beiden Monitorings teilnahmen, können so zudem einen Überblick über die Veränderungen in ihrem Spital gewinnen.

\section{Rücklauf und Stichprobe}

Das Monitoring-Instrument zum kRM wurde an die CEOs aller Schweizer Spitäler versandt mit der Bitte, es an die zuständige Person für kRM weiterzuleiten. Der Befragungszeitraum war April-Juli 2010. Wie schon beim ersten Monitoring lag die Rücklaufquote der Erhebung bei $43 \%$ (138 von 321 Spitälern). $2 / 3$ der Spitäler machten zum zweiten Mal mit und $1 / 3$ der Spi-

\section{Seconde enquête suisse sur la gestion des risques cliniques dans l'hôpital}

En été 2010 le centre pour les sciences de l'organisation et du travail de I'EPF Zurich a réalisé la seconde enquête suisse sur la gestion des risques cliniques (GRC) dans les hôpitaux suisses. Comme à la première enquête en $2007 / 2008$ y ont participé $43 \%$ des hôpitaux suisses. Les responsables de la GRC ont reçu fin 2010 une évaluation détaillée spécifique de leur hôpital sur toutes les questions de l'enquête. Celle-ci donne une vue d'ensemble de la situation actuelle ainsi que des développements prévues dans le domaine de la GRC dans les hôpitaux suisses qui ont participé.

Les changements les plus évidents comparé à I'enquête $2007 / 2008$ sont une systématisation croissante de la GRC ainsi qu'un meilleur encadrement du thème dans l'organisation (par ex. augmentation de personnes responsables et définition des buts stratégiques pour la GRC). Ce développement correspond aux suggestions de la première enquête et souligne encore I'utilité des activités dans le cadre des enquêtes. En même temps, les personnes responsables pour la GRC dans les hôpitaux considèrent toujours un grand potentiel d'optimisation dans la GRC.

täler nahm zum ersten Mal teil. In der Deutschschweiz antworteten 94 von 217 angeschriebenen 
Tabelle 1

Rücklauf 2010 in absoluten Zahlen bzw. in Prozent nach Spitaltyp.

\begin{tabular}{llllllr|} 
Spitaltyp & $\begin{array}{l}\text { Universitäts- } \\
\text { spitäler }\end{array}$ & $\begin{array}{l}\text { Akut- } \\
\text { spitäler }\end{array}$ & $\begin{array}{l}\text { Psychiatrische } \\
\text { Kliniken }\end{array}$ & $\begin{array}{l}\text { Rehabilitations- } \\
\text { kliniken }\end{array}$ & $\begin{array}{l}\text { Spezial- } \\
\text { kliniken* }\end{array}$ & \multicolumn{1}{l}{ Total } \\
\hline Teilnahme & $5(100 \%)$ & $78(45 \%)$ & $23(48 \%)$ & $13(30 \%)$ & $19(37 \%)$ & $138(43 \%)$ \\
\hline $\begin{array}{l}\text { Absage** } \\
\text { Keine Rück- } \\
\text { meldung }\end{array}$ & 0 & $48(28 \%)$ & $11(23 \%)$ & $8(19 \%)$ & $15(29 \%)$ & $82(26 \%)$ \\
Total & 5 & $47(27 \%)$ & $14(29 \%)$ & $22(51 \%)$ & $18(35 \%)$ & $101(31 \%)$ \\
& & 173 & 48 & 43 & 52 & 321
\end{tabular}

* Spezialkliniken waren beim ersten Monitoring unter Akutspitäler subsummiert.

** Absagen erfolgten in den meisten Fällen wegen Zeitmangels oder weil das Spital zu klein sei oder sich das kRM erst im Aufbau befinde.

Spitälern (43\%; 2007/2008: 46\%), in der französischsprachigen Schweiz 35 von 86 (41\%; 2007/2008: $30 \%)$ und in der italienischsprachigen Schweiz 9 von 18 (50\%; 2007/2008: 67\%). Manche Spitäler beantworteten den Erhebungsbogen als Spitalgruppe oder -verbund, so dass schliesslich Daten von 98 verschiedenen Spitälern oder Spitalgruppen vorlagen, die insgesamt die Situation in 138 Spitälern abbildeten.

Tabelle 1 zeigt den Rücklauf nach Spitaltyp. Die im Folgenden ausgewählten Ergebnisse beziehen sich jeweils auf alle antwortenden Spitäler. Sie berücksichtigen alle Daten aus 2010, also nicht nur diejenigen der Spitäler, die an beiden Erhebungen teilgenommen haben.

\section{Organisationale Einbettung und Ressourcen des KRM}

\section{Beschreibung des kRM-Teams}

63\% der Personen, die den Erhebungsbogen ausgefüllt hatten, arbeiteten länger als 5 Jahre im entsprechenden Spital. Die Funktion einer zentralen Koordination des kRM existierte zum Zeitpunkt der Befragung in $65 \%$ der antwortenden Spitäler, was eine in der Linie der Spitalleitung verankert. Die für kRM zur Verfügung stehenden Personalressourcen waren nach wie vor eher gering: Der Median für das zentrale kRM zeigte $280 \%$ (Universitätsspitäler), 20\% (Akutspitäler und Psychiatrische Kliniken) und 7,5-10\% Stellenprozente (Reha-, Spezial-Kliniken). Hinzu kamen dezentrale (z.B. in den einzelnen Kliniken vorhandene) Personalressourcen für kRM in ähnlichem Umfang. Über ein institutionalisiertes Forum zum Austausch mit einzelnen Kliniken/Fachbereichen verfügten $55 \%$ der antwortenden Spitäler (geplant in weiteren 13\%).

\section{Strategische Verankerung des kRM}

Über eine verbindliche, schriftlich festgelegte Strategie verfügten $69 \%$ (2007/2008: 58\%) der antwortenden Spitäler. 41\% (2007/2008: 34\%) besassen verbindliche, schriftlich festgelegte strategische Ziele spezifisch für das kRM (in 16\% der Spitäler geplant). Als wichtigste strategische Ziele wurden $\mathrm{u}$.a. Aufbau, Verbesserung und Systematisierung des kRMs, Verbesserungen und Standardisierungen der Prozesse und Sensibilisieren und Befähigen der Mitarbeitenden genannt.

\section{Von den antwortenden Spitälern verfügten $41 \%$ über verbindliche, schriftlich festgelegte Ziele für kRM.}

klare Zunahme um 16\% gegenüber 2007/2008 darstellt. 16\% (2007/2008: $24 \%)$ der Spitäler planten nach eigenen Angaben, eine solche Funktion in den nächsten 6-12 Monaten einzuführen, bei 19\% (2007/ 2008: $27 \%$ ) war dies nicht vorgesehen. Die meisten Spitäler führten eine solche Funktion seit dem Jahr 2000 und jetzt ein (das erste Spital bereits 1991).

Bezüglich organisationaler Einbettung der kRMFunktion zeigte sich in 67\% (2007/2008: 73\%) der Spitäler ein enger Bezug zur Spitalleitung. 30\% der kRM-Verantwortlichen waren Mitglied der Spitalleitung, 25\% hatten Stabsstellen inne und 12\% waren
Wie sich in Interviews begleitend zum Monitoring 2007/2008 zeigte, wird ein guter Zugang zu allen im Spital tätigen Berufsgruppen für die strategische Verankerung des kRM als zentral angesehen, da sonst die Umsetzung im Alltag erschwert wird. Das Monitoring zeigte einen breiten Einbezug der unterschiedlichen Berufsgruppen in den kRM-Teams. 2010 am häufigsten vertreten waren diplomierte Pflegefachleute (in 59\% der Spitäler, in 15\% mit Studium der Pflegewissenschaften), Personen mit Medizin-Studium (in 54\% der Spitäler, die meisten Personen mit Facharzttitel FMH) und jeweils in 23\% der Spitäler 
Personen mit medizinisch-technischer oder medizinisch-therapeutischer Ausbildung. Es fanden sich Personen mit betriebswirtschaftlicher (in 66\% der Spitäler), psychologischer (21\%) und juristischer (7\%) Aus-/Weiterbildung. Im Qualitätsmanagement weitergebildet waren Personen in 70\% (Ausbau in $6 \%$ geplant) und im $\mathrm{kRM}$ in $36 \%$ (Ausbau in $8 \%$ geplant) der Spitäler.

\section{Entwicklungsstand des kRM}

Auch 2010 erwies sich der Entwicklungstand des kRM bei den teilnehmenden Spitälern als heterogen. Die Antworten auf die meisten Fragen fielen jedoch insgesamt systematischer als 2007/2008 aus.

\section{Risikomanagementprozess und weitere Aspekte des KRM auf Spitalebene}

Die aktuelle Situation des kRM auf Spitalebene wurde anhand von 15 Fragen entlang eines systematischen Risikomanagementprozesses [2,5] und weiterer erfolgskritischer Faktoren erfasst. Abbildung 1 zeigt ausgewählte Resultate im Vergleich zu den Antworten 2007/2008.

Ähnlich viele Spitäler wie 2007/2008 gaben eine mindestens unsystematische Umsetzung bei eher reaktiven Massnahmen an. So analysierten $76 \%$ der Spitäler (44\% systematisch, 32\% unsystematisch) die Ursachen und Umstände eines kritischen Ereignisses oder Fehlers im Behandlungsprozess auf Spitalebene. Noch mehr (42\% systematisch, $40 \%$ unsystematisch) gaben an, aufgrund der Ursachenanalyse eines kritischen Ereignisses oder Fehlers im Behandlungsprozess auf Spitalebene Massnahmen abzuleiten. Deutlich mehr Spitäler (64\%; 2007/2008: 51\%) hatten zumindest unsystematische Definitionen von Aufgaben, Kompetenzen und Verantwortlichkeiten im kRM. Von allen Aspekten des Risikomanagementprozesses waren spitalweite Berichte zum kRM wiederum am wenigsten verbreitet (in $48 \%$ der Spitäler; 2007/2008: 28\%). 14\% (2007/2008: 18\%) planten

\section{Abbildung 1}

Ausgewählte Ergebnisse zur aktuellen Situation des kRM im Spital 2010 und 2007/2008.

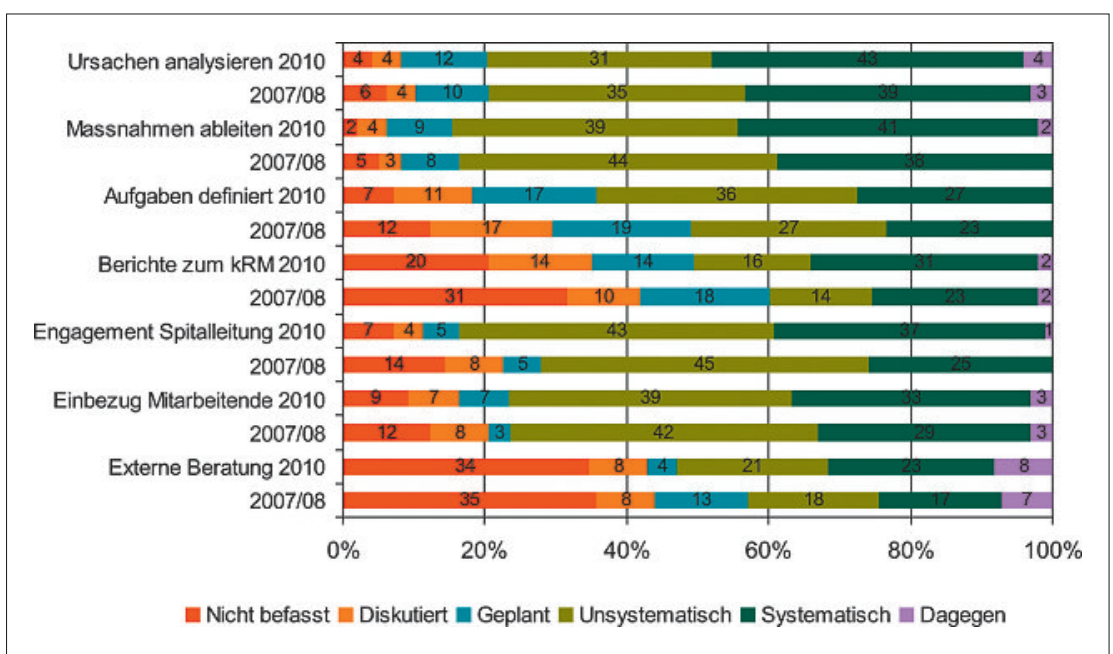

die Umsetzung für die nächsten 12 Monate, weitere $14 \%$ (10\%) diskutierten dies, aber ohne Umsetzungspläne zu haben, und $21 \%(32 \%)$ hatten sich noch nicht damit befasst.

Weitere Aspekte des kRM auf Spitalebene betrafen u. a. die Führung, Partizipation und Ausbildung. Wiederum wurden die Fragen «Die Spitalleitung macht durch konkrete Aktivitäten ihr Engagement bei Fragen zur Patientensicherheit sichtbar» (82\%; 2007/ 2008: $72 \%$ ) und «Die Mitarbeitenden werden aktiv ins kRM einbezogen (z. B. bei der Identifikation klinischer Risiken)» (73\%; 2007/2008: 73\%) am meisten als «systematisch bzw. unsystematisch umgesetzt» beantwortet. Am wenigsten (45\%; 2007/2008: 36\%) Spitäler gaben an, dass externe Stellen in die Weiterentwicklung des kRM eingebunden werden (z.B. durch Beratungen, Audits usw.); $8 \%$ der Spitäler entschieden sich hier «bewusst dagegen».

\section{Meldesysteme für kritische Ereignisse (Incident Reporting)}

Von den antwortenden Spitälern verfügten 2010 71\% über ein spitalweites Incident Reporting System (IRS), bei weiteren $9 \%$ war es in einzelnen Kliniken umgesetzt und in $13 \%$ in der Einführung bzw. geplant. Am häufigsten wurde das IRS zwischen 2004-2007 eingeführt, vorher hatten erst 14 Spitäler eines. $6 \%$ der Spitäler verfügten über kein IRS.

Die Umsetzung war systematischer als für andere Bereiche des kRM. So wurden als mehrheitlich systematisch umgesetzt angegeben: Definition von zu meldenden Ereignissen ist vorhanden (77\%), Einführungen/Schulungen finden statt (63\%), Massnahmen werden aus gewonnenen Erkenntnissen abgeleitet (66\%) und den Mitarbeitenden kommuniziert (64\%). $47 \%$ der Spitäler analysierten die Ursachen eines kritischen Ereignisses systematisch mit einer standardisierten Vorgehensweise und $45 \%$ überprüften die umgesetzten Massnahmen systematisch. In $33 \%$ der Spitäler wurden Schadensfälle bewusst nicht ins IRS miteinbezogen, $14 \%$ hatten ein nicht-anonymes und $7 \%$ ein nicht-elektronisches IRS.

Die Antworten zu den offenen Fragen im Monitoring-Instrument zeigten, dass IRS nicht mehr wie 2007/2008 das dominierende Thema war. Offensichtlich hatten viele Spitäler inzwischen schon ein paar Jahre Erfahrung mit einem IRS. Aktuell standen häufiger Fragen der Systematisierung des kRM und der Umsetzung des RM-Prozesses (Risikoerfassung, -bewertung usw.) im Vordergrund.

\section{Optimierungspotential im kRM}

Trotz höherer Systematisierung sahen die Antwortenden das Optimierungspotential im kRM als unverändert hoch an. Dieses Resultat bestätigt die Annahme von 2007/2008, dass fast unabhängig vom tatsächlichen Entwicklungsstand ein hoher Anspruch bezüglich kRM zu einer hohen Einschätzung des Optimierungspotentials führt. Auch diesmal standen die Fort- 
bildung zu Themen der Patientensicherheit (83\% mehrheitliche oder volle Zustimmung) und die Standardisierung von Verfahren und Prozessen (78\% Zustimmung) an der Spitze. Fast ebenso gewünscht wurden klarere Regelungen der Aufgaben, Kompetenzen und Verantwortlichkeiten sowie ein offenerer und ehrlicherer Umgang mit Fehlern und Schwachstellen (je 70\% Zustimmung) und ein regelmässigerer Austausch zwischen dem zentralen kRM und einzelnen Kliniken (68\% Zustimmung). Zusätzliche finanzielle oder personelle Ressourcen wurden in fast $50 \%$ als notwendig erachtet, um die Wirksamkeit des kRM zu erhöhen.

\section{Anmerkungen und Ausblick}

Die Ergebnisse des zweiten Monitorings bilden den nationalen Stand $2010 \mathrm{im}$ kRM ab. Da die Teilnahme für die Spitäler freiwillig war, sind die Ergebnisse nicht repräsentativ und können nur auf die $43 \%$ antwortenden Spitäler bezogen werden. Es ist zu vermuten, dass die Resultate ein eher zu positives Bild zeigen und einige Spitäler sich erst im Aufbau des kRM befinden oder sich mit der Thematik noch wenig auseinandergesetzt haben. Trotzdem verdeutlicht der wiederum hohe Rücklauf die Wichtigkeit des Themas und die Akzeptanz des Monitorings. Die Resultate zeigen eine zunehmende Systematisierung des kRM und eine vermehrte organisationale Verankerung des Themas (z.B. Zunahme von für kRM verantwortlichen Personen und strategischen Zielen für kRM). Diese Entwicklung entspricht den Empfehlungen des ersten Monitorings und unterstreicht den Nutzen solcher Monitoring-Aktivitäten. Des Weiteren fällt auch auf, dass Entwicklungen und Umsetzung Zeit brauchen und von den verantwortlichen Personen nach wie vor viel Optimierungspotential gesehen wird.

\section{Die Resultate zeigen eine zunehmende Systematisie- rung des klinischen Risikomanagements und eine vermehrte organisationale Verankerung des Themas.}

Ein Blick in die Literatur zeigt, dass es Studien zu Qualitätsmanagement und einzelnen Elementen des kRM in verschiedenen Ländern gibt [6, 7], ein nationales Monitoring zum kRM oder auch Patientensicherheit jedoch bisher international fehlte [8]. Neben dem hier vorgestellten Monitoring in der Schweiz führte das Institut für Patientensicherheit Bonn ebenfalls im Jahr 2010 die erste bundesweite Krankenhausbefragung zum kRM in Deutschland durch (www.ifpsbonn.de/projekte-1/projekte/projektkrankenhausbefragung-und-sicherheitskultur). Der dabei eingesetzte Fragebogen übernahm viele Fragen aus dem in dieser Studie verwendeten MonitoringInstrument, was Möglichkeiten für vergleichende Analysen eröffnet.
Reaktionen auf die internationale Publikation zum Monitoring-Instrument [2] und die Zusammenarbeit mit dem Institut für Patientensicherheit Bonn eröffnen interessante Perspektiven, was das kRMMonitoring betrifft. Für die ETH und Hochschule Luzern als Forschungseinrichtungen ist die Entwicklungsarbeit bald abgeschlossen und kein erneutes Monitoring in Planung. Die Frage, wie das Monitoring operativ weitergeführt wird, ist noch offen. Jedenfalls ist ein kontinuierliches Monitoring des kRM in regelmässigen Abständen wichtig, um vergleichbare Transparenz zu gewinnen und Entwicklungen zu messen und um das Bewusstsein für Risikomanagement als kontinuierlichen Prozess zu fördern.

\section{Danksagung}

Das zweite kRM-Monitoring wurde finanziert vom Zentrum für Organisations- und Arbeitswissenschaften der ETH Zürich und unterstützt von der Hochschule Luzern - Wirtschaft. Als Folgeprojekt baute es auf das Projekt «Klinisches Risikomanagement in Schweizer Spitälern (KTI 8614.1 ESPP-ES)» auf. Ein besonderer Dank geht an Sandra De Pasquale, Andrea Eller und Fabian Burri sowie an die Mitglieder des Projektteams: Tanja Manser, Oliver Kessler, Yvonne Pfeiffer und Theo Wehner.

\section{Literatur}

1 Manser T, Kessler O, Briner M, Pfeiffer Y, Wehner T. Klinisches Risikomanagement in Schweizer Spitälern. Projektbeschreibung, Ablauf und Ausblick. Schweiz Ärztezeitung. 2007;88(51/52):2168-9.

2 Briner M, Kessler O, Pfeiffer Y, Wehner T, Manser T. Assessing hospitals' clinical risk management. Development of a monitoring instrument. BMC Health Services Research. 2010;10:337.

3 Briner M, Kessler O, Pfeiffer Y, Wehner T, Manser T. Erste Schweizer Erhebung zum klinischen Risikomanagement im Spital. Zusammenfassung der Ergebnisse. Schweiz Ärztezeitung. 2009;90(15/16):635-8.

4 Middendorf C. Klinisches Risikomanagement. Implikationen, Methoden und Gestaltungsempfehlungen. Münster: LIT;2005.

5 Kahla-Witzsch HA. Praxis des Klinischen Risikomanagements. Landsberg/Lech: ecomed Medizin;2005.

6 Lombarts MJ, Rupp I, Vallejo P, Klazinga NS, Sunol R. Differentiating between hospitals according to the «maturity» of quality improvement systems: A new classification scheme in a sample of European hospitals. Quality \& safety in health care. 2009;18(Suppl 1):i38-43.

7 Makai P, Klazinga N, Wagner C, Boncz I, Gulacsi L. Quality management and patient safety: survey results from 102 Hungarian hospitals. Health policy. 2009;90(2-3):175-80

8 Leape LL, Berwick DM. Five years after To Err Is Human: What have we learned? Journal of the American Medical Association. 2005;293(19): 2384-90.

9 Pfeiffer Y, Manser T, Wehner T. Conceptualising barriers to incident reporting. A psychological framework. Quality and Safety in Healthcare. 2010;19:e60. 